\title{
Film Stiffness Analysis for Angular Contact Ball Bearings Considering Thermal Effect
}

\author{
Chun L. Lei, Zhi Y. Rui, Qin Wu, Jun F. Guo and Li N. Ren
}

Key Laboratory of Digital Manufacturing Technology and Application, The Ministry of Education, Lanzhou University of Technology, Lanzhou, China; School of Mechanical \& Electronic Engineering, Lanzhou University of Technology, Lanzhou, China

\begin{abstract}
In order to more accurately calculate the film stiffness of angular contact ball bearing, it is necessary to establish the film stiffness calculation model that is consistent with reality. The frictional heat exists in high-speed ball bearings, and can impact on oil film thickness and stiffness. The calculation model of film stiffness of an angular contact ball bearing taking account of the effects of viscous heating was proposed based on the elastohydrodynamic lubrication theory. The central film thickness and film stiffness have been determined. An example was calculated with this derived equation and the result was compared with that given in other literatures. The calculation results show that the central film thickness decreases and the film stiffness increases when friction heating are considered.
\end{abstract}

Keywords: Ball bearing, Elastohydrodynamic lubrication, Film stiffness, Heat effects.

\section{INTRODUCTION}

As the key component of rotating machinery, bearingrotor system plays a more and more important role in the industrial field. The support stiffness of a bearing has a great influence on the precision and dynamics performance of rotating machinery. To calculate the stiffness of bearing considering thermal effects, it is necessary to analyze the actual working conditions of bearing and the detailed loading parameters, including the load, the speed, the lubricating film thickness and the viscosity, and so on.

In previous work, great efforts have been made by researcher to develop a computing method of bearing stiffness. However, the existence of oil film and its impact on the stiffness are ignored, and the contact between rolling elements and raceways is regarded as pure Hertz contact by approximate method. Wang [1] calculated the contact stiffness and synthesizing stiffness of angular contact ball bearing, which included the centrifuge displacement of the inner raceway. Actually, the integral rigidity of rolling bearing is composed of the contact stiffness and film stiffness. With the development of computational techniques, based on elastohydrodynamic lubrication, the studies of influence of stiffening effect of lubricating film on bearing dynamics characteristics were taken into account [2,3]. Considering film thickness, the axial stiffness formula of tapered roller bearing was derived, and also explained that the existence of oil film had significant impact on bearing stiffness by using an example [4-6]. Koye and Winer [7] compared their optical film measurement and the calculated film thickness by

*Address correspondence to this author at the Key Laboratory of Digital Manufacturing Technology and Application, The Ministry of Education, Lanzhou University of Technology, 287 Langongping Road, Qi Li He, Lanzhou, Postcard: 730050, China; Tel: +86 9312975 705;

E-mail: lclyq2004@163.com isothermal theory, and the results showed a good accordance between them. In fact, the frictional heat of ball bearing can be generated under high speed conditions. However, the influence of viscosity shear heat of oil film on film stiffness is not considered in most models mentioned above. Cheng $[8,9]$ presented the most comprehensive model of the elastohydrodynamic lubrication process. Not only the viscous heating but also heat transfer within the lubricant film and the bearing surface are considered in his work. He draws a conclusion that the viscous heating in the inlet zone could substantially reduce the film thickness. However, the influence of the lubricant was only manifested at very high surface speeds. In this paper, based on elastohydrodynamic lubrication theory, the film thickness between ball and raceways is analyzed. Considering the impact of thermal effects on film thickness, the calculation model of film stiffness of angular contact ball bearing is proposed to be in agreement with actual conditions.

\section{GOVERNING EQUATION}

A two-dimensional isothermal Reynolds equation for elliptical contact could be written as

$$
\begin{aligned}
& \frac{\partial}{\partial x}\left(\frac{h^{3}}{\partial x} \frac{\partial p}{\partial x}\right)+\frac{\partial}{\partial y}\left(\frac{h^{3}}{\frac{\partial p}{\partial y}}\right) \\
& =12 U \frac{\partial}{\partial x}(h)+12 V \frac{\partial}{\partial y}(h)
\end{aligned}
$$

where $\rho$ is the lubricating density, $p$ is the local pressure, $h$ is the thickness of film, and $\eta$ is the fluid viscosity. The parameter $U$ and $V$ are the average velocity of bearing lubricant in the $x$ and $y$ directions respectively. $U=\left(u_{1}+u_{2}\right) / 2$, $V=\left(v_{1}+v_{2}\right) / 2$. 
The elastic deformation equation is given to describe the deformation displacement of each point on the elasticity surface:

$$
(x, y)=\frac{2}{E^{\prime}} \iint \frac{p(,)}{\sqrt{(x)^{2}+(y \quad)^{2}}} d d
$$

where and are, respectively, the addition coordinate corresponding to the $x$ and $y$ directions. is the solution domain. $E^{\prime}$ is the equivalent elastic modulus. by

The film thickness at any point in the contact zone is given

$$
h(x, y)=h_{0}+\frac{x^{2}}{2 R_{x}}+\frac{y^{2}}{2 R_{y}}+(x, y)
$$

where $h_{0}$ is the minimum lubricant film thickness. $R_{x}$ and $R_{y}$ are an equivalent radius of curvature along the $x$ and $y$ directions, respectively.

\section{FILM STIFFNESS MODEL OF A BALL BEARINGS}

As discussed in the above section, according to these governing equations, the film thickness and distribution of angular contact ball bearing can be obtained at isothermal condition. Hamrock and Dowson [10] established a generalized formula for central lubricant film thickness in an elliptical area point contact, which is adopted in this article. The equation is

$$
H_{c}=2.69 \frac{U^{0.67} G^{0.53}}{W^{0.067}}\left(\begin{array}{ll}
1 & 0.61 e^{0.73 k}
\end{array}\right)
$$

where:

$$
\begin{aligned}
& H_{c}=\frac{h_{c}}{R_{x}} \\
& G=E \\
& U=\frac{{ }_{0} U}{E R_{x}} \\
& W=\frac{W}{E R_{x}^{2}}
\end{aligned}
$$

$k=a / b$

$G, U, W$ are dimensionless parameters. ${ }_{0}$ is the fluid viscosity at atmospheric pressure $(\mathrm{Pa} \cdot \mathrm{s})$; $\quad$ is the pressure coefficient of viscosity; $W$ is the load acting on roller. $k$ is the ellipticity.

Obviously, the influence of the frictional heat on film thickness was not considered in the formula by Hamrock and Dowson. When the bearing runs at high speeds, some of the frictional heat generated in each concentrated contact region is dissipated in the lubricant momentarily residing in the contact inlet zone. This effect tends to increase the temperature of the lubricant in the contact region. Because the big velocity gradient in oil film due to high speed can give rise to viscosity shear heat, moreover, if the roller is scrolling with sliding, shear heat will exist in whole lubrication area, which can change the temperature of lubricant films. The results by Cheng [11] showed that the sliding in contact zones had little influence on the central film thickness and could be disregard. Meanwhile, according to the experimental result by Crook, the film thickness mainly depended on lubricant viscosity in entrance region, that is, the film thickness relied on the temperature in its initial entrance zone. So, the approximate calculation method developed by Грубин [12] is reasonable.

According to Грубин formula, assuming $H_{0, l}=h_{0} / R_{x}$ is the entrance film thickness parameter at the isothermal condition, and $H_{0, T}$ is the entrance film thickness parameter considering heat effects, the relationship between the two is given by

$H_{0, T}=c H_{0, l}$

where $c$ is the heat correction factor, which can reflect the influence of shear heat in the inlet zone on film thickness, the equation is

$c=\frac{3.94}{3.94+L^{0.62}}$

where

$L={ }_{0} U^{2} / K ; K$ is the coefficient of heat conduction of lubricating oil; is the coefficient for calculating viscosity as a function of temperature. At the same time, the numerical calculation results by Dowson showed that when ellipticity $\mathrm{k}>5$, the film thickness $h_{0}$ in entrance zone calculated by Грубин formula was identified with the central film thickness $h_{c}$ in an elliptical area point contact calculated by Hamrock and Dowson. So, by substituting Eqs. (4) and (11) into Eq. (10), the film thickness parameter formula is determined from

$$
H_{0, T}=2.69 \frac{U^{0.67} G^{0.53}}{W^{0.067}} \nsucc\left(1 \quad 0.61 e^{0.73 k}\right) \times \frac{3.94}{3.94+L^{0.62}}
$$

Then, by substituting Eq. (12) into Eq. (5), the central film thickness calculation formula considering the influence of temperature rise is obtained.

$$
\begin{aligned}
& h_{c}=2.69 \frac{U^{0.67} G^{0.53}}{W^{0.067}}\left(\begin{array}{ll}
1 & 0.61 e^{0.73 k}
\end{array}\right) \\
& \times \frac{3.94}{3.94+L^{0.62}} \times R_{x}
\end{aligned}
$$

According to the definition of stiffness, the expression for central film stiffness in the contact zone considering heat effects is given by

$$
\begin{aligned}
& k_{o}=\lim _{h \rightarrow 0} \frac{W}{h_{c}}=\frac{d W}{d h_{c}} \\
& =2.5955 \times 10^{9} h_{c}{ }^{15.9254} U^{10} G^{7.9104} E^{\prime} R_{x}^{16.9254} \\
& \text { (1 } \left.10.61 e^{0.73 k}\right)^{14.9254} \cdot\left(\frac{3.94}{3.94+L^{0.62}}\right)^{14.9254}
\end{aligned}
$$

Zhao [13] recommended that the film rigidity at Hertz contact zone was equivalent to the derivative of the force in 
the zones of contact versus the central film thickness, the formula of film stiffness in the contact zones was defined as:

$k_{c}=\frac{\partial W}{\partial h_{c}}=\frac{10}{1.8023} E^{\prime} R_{x}$

$\cdot\left[U^{0.67} G^{0.53} W^{1.067}\left(\begin{array}{ll}1 & 0.61 e^{0.73 k}\end{array}\right)\right]^{1}$

\section{RESULTS AND DISCUSSIONS}

According to experimental condition by Fan [14], the film stiffness considering heat effects is calculated. The experimental parameters are given as follows: the equivalent radius of curvature $R_{x}$ is $0.0127 \mathrm{~m}$; the equivalent elastic modulus $E$ is $1.17 \times 10^{11} \mathrm{pa}$; the fluid viscosity at atmospheric pressure ${ }_{0}$ is $117.0 \mathrm{~Pa} \cdot \mathrm{s}$; the pressure coefficient of viscosity is $3.0 \times 10^{-8} \mathrm{~m}^{2} / \mathrm{N}$; the coefficient for calculating viscosity is $0.042 / \mathrm{K}$; the average entrainment velocity of lubricating oil $U$ is $387 \times 10^{-6} \mathrm{~m} / \mathrm{s}$. The calculation result using the method presented in this article is compared with that by Eq. (15). The results are shown in Table $\mathbf{1 .}$

Table 1. Film stiffness of isothermal EHL and thermal EHL.

\begin{tabular}{|c|c|c|}
\hline $\begin{array}{c}\text { Loading } \\
(\mathbf{N})\end{array}$ & $\begin{array}{c}\text { Film Stiffness of } \\
\text { Isothermal EHL }\left(\times \mathbf{1 0}^{\mathbf{8}} \mathbf{~} / \mathbf{m}\right)\end{array}$ & $\begin{array}{c}\text { Film Stiffness of } \\
\text { Thermal EHL }\left(\times \mathbf{1 0}^{\mathbf{8}} \mathbf{~} / \mathbf{m}\right)\end{array}$ \\
\hline \hline 8 & 1.9412 & 1.9413 \\
\hline 9.5 & 2.3318 & 2.3320 \\
\hline 11 & 2.7267 & 2.7269 \\
\hline 12.5 & 3.1251 & 3.1254 \\
\hline 14 & 3.5268 & 3.5271 \\
\hline 15.5 & 3.9314 & 3.9318 \\
\hline 17 & 4.3387 & 4.3390 \\
\hline 18.5 & 4.7483 & 4.7487 \\
\hline 20 & 5.1602 & 5.1606 \\
\hline 21.5 & 5.5742 & 5.5746 \\
\hline 23 & 5.9901 & 5.9906 \\
\hline 24.5 & 6.4078 & 6.4083 \\
\hline
\end{tabular}

From Table 1, at high speed, under the same conditions of loading, the film stiffness considering heat effects is bigger than that without heat effects. Because the film thickness at Hertz contact zone depends on the flow of lubricant through entrainment movement in the inlet zone, and the different velocity of oil film can lead to the viscous shear, so it can increase the temperature of the lubricant. The lubricant film thickness decreases as a result of temperature increase in the contact region, so that the film stiffness increases.

\section{CONCLUSION}

In this paper, the film stiffness model of angular contact ball bearing considering heat effects is presented. The main objectives are to calculate the film stiffness more accurately to obtain the comprehensive bearing rigidity model and the following conclusions can be obtained:

(1) The frictional heat has great influence on film stiffness, and cannot be neglected in the actual calculations.

(2) The film stiffness considering heat effects is higher than that without heat effects, and the film stiffness decreases with increasing speed.

\section{CONFLICT OF INTEREST}

The authors confirm that this article content has no conflict of interest.

\section{ACKNOWLEDGEMENTS}

This project is supported by National Natural Science Foundation of China (Grant No. 51465035), the Program for Changjiang Scholars and Innovative Research Team in University of Ministry of Education of China (Grant No. IRT1140) and Hongliu young teacher preparation programs of Lanzhou University of Technology (Grant No. Q201212).

\section{REFERENCES}

[1] B. M. Wang, X. S. Mei, C. B. Hu, Z. X. Wu, "Influence of inner ring centrifugal displacement on high speed angular contact ball bearing stiffness", Chin. J. Computat. Mech., vol. 27, no. 1, pp. 145-150, 2010

[2] L. C. Zhao, J. J. Ma, C. Q. Ma, Z. R. Qiu, "Effect of lubrication on the vibration characteristics of ball bearings", Tribology, vol. 23, no. 5, pp. 421- 425, 2003.

[3] M. X. Wu, W. Wu, J. B. Hu, S.H.Yuan, C. Wei, "Oil film stiffness calculation of high speed angular contact ball bearing considering spinning", J. Vib. Shock, vol. 33, no. 10, pp. 38- 42, 2014.

[4] H. Wu, Research on the Dynamic Characteristics of Rolling Element Bearing and the Dynamic Model of Bearing Rotor System, Shanghai: East China University of Science and Technology, 2010.

[5] H. Hao, P.S. Zhang, H.Y. Yu, J.M. Wen, "Study on the influence of elastohydrodynamic lubrication on bearing stiffness in angular contact bearing”, Comput. Eng., vol. 25, no. 11, pp. 2-3, 1999.

[6] Y. Tang, G. Luo, J. Zhang, and D. P. Gao, "Theoretical analysis and experiment of the high speed ceramic rolling bearing equivalent stiffness", J. Aerospace Power, vol. 20, no. 2, pp. 240-244, 2005.

[7] K. A. Koye, and W. O. Winer, "An experimental evaluation of the Hamrock and Dowson minimum film thickness equation for fully flooded ehd point contacts", Trans. ASME, vol. 103, no. 4, pp. 284294, 1981.

[8] H. S. Cheng, Prediction of film thickness and sliding frictional coefficient in elastohydrodynamic contacts, Des Eng Tech Conf,, New York City, NY, USA, October5-9, vol. 1, pp. 285-293, 1974.

[9] H. S. Cheng, "Isothermal elastohydrodynamic lubrication theory in the full range of pressure viscosity coefficient transaction of the ASME", J. Lubricat. Technol., vol. 94, no. 1, pp. 35-43, 1972.

[10] B. J. Hamrock, and D. Dowson, "Isothermal elasto-hydrodynamic lubrication of point contacts, part I theoretical formulation, Transaction of the ASME", J. Lubricat. Technol., no. 98, pp. 223229, 1976. 
[11] H. S. Cheng, "A refined solution to the thermal-elastohydrodynamic lubrication of rolling and sliding cylinders", Trans. ASLE, vol. 8 , no. 2 , pp. 397-410, 1965

[12] Z. Wen, Principles of Tribology, Tsinghua University Press, pp. 277312, 1990.
[13] L. Zhao, Research on Vibration of Ball Bearing, Hangzhou: Zhejiang Univ., 2003

[14] B. Fan, F. Guo, X. Li, "Experimental study of elastohydrodynamic lubrication films under spinning/rolling conditions", Lubricat. Eng., vol. 33, no. 11, pp. 46-50, 2008.

(C) Lei et al.; Licensee Bentham Open.

This is an open access article licensed under the terms of the Creative Commons Attribution Non-Commercial License (http://creativecommons.org/licenses/by-nc/3.0/) which permits unrestricted, non-commercial use, distribution and reproduction in any medium, provided the work is properly cited. 